\title{
OC-116 THE UK EXPERIENCE OF LIVER TRANSPLANTATION IN PATIENTS RECEIVING OPIATE REPLACEMENT THERAPY
}

doi:10.1136/gut.2011.239301.116

B J Hogan, ${ }^{1 *}$ E Shepherd, ${ }^{1}$ I Webzell, ${ }^{2} \mathrm{~K}$ Webb, ${ }^{3} \mathrm{~K}$ Agarwal, ${ }^{2} \mathrm{M}$ Aldersley, ${ }^{4} \mathrm{~A}$ Bathgate, ${ }^{5} \mathrm{~J}$ Ferguson, ${ }^{3}$ W J H Griffiths, ${ }^{5}$ M Hope, ${ }^{5} \mathrm{~J}$ Wood, ${ }^{6}$ S Masson, 7 K Rothwell, ${ }^{4}$ D Thorburn' ${ }^{1}$ Shelia Sherlock Liver Centre, Royal Free Hospital, London, UK; ${ }^{2}$ Institute of Liver Studies, Kings College Hospital, London, UK; ${ }^{3}$ Department of Hepatology, Queen Elizabeth Hospital, Birmingham, UK; ${ }^{4}$ Department of Hepatology, St James University Hospital, Leeds, UK; ${ }^{5}$ Department of Hepatology,,${ }^{6}$ Addenbrookes Hospital, Cambridge, UK; ${ }^{7}$ Department of Hepatology, Freeman Hospital, Newcastle upon Tyne, UK

Introduction There is limited outcome data on patients undergoing liver transplantation (LT) while receiving opiate replacement therapy (ORT). In 2008 the UK transplant centres adopted consensus guidelines for the assessment and management of patients on ORT prior to LT. We report the collated UK experience of LT in patients receiving ORT.

Methods All UK transplant centres submitted retrospective data, using a standardised proforma.

Results 75 LT assessments were undertaken between 2001 and 2010 in patients using ORT, 36 pre and 39 post introduction of national guidelines. 51/75 (68\%) were listed. Detailed information on aetiology was available for 44 patients listed. 38/44 (86\%) were male, median age was 47 (32-61). Aetiology of liver disease was Hepatitis C virus (HCV) in 27/44 (61\%), HCV and alcohol in 14/44 (32\%) and alcohol in 3/44 (7\%). $13 / 44$ patients had hepatocellular carcinoma. Pre-2008 14/28 $(50 \%)$ underwent formal substance misuse assessment prior to listing, compared to $13 / 14$ (93\%) post-2008. 42/44 patients 
injected (39/42 heroin) and 2/44 inhaled/smoked illicit drugs for a median duration of 15 years. The median abstinence from illicit drug use and duration of ORT were both 60 months. ORT was methadone in 40/44, buprenorphine $2 / 44$, dihydrocodeine $1 / 44$ and methadone combined with diamorphine (1/44). The median dose of methadone was $40 \mathrm{mg}$ at assessment and 50mg after LT. 29/51 (57\%) listed received LT, $12 / 51$ (24\%) died or deteriorated, 5/44 (11\%) were delisted for substance misuse, 1/51 improved and was removed from the list and 4/51 remain active. Median ITU and total hospital stay was 2 and 16.5 days respectively. Overall survival after LT was $86 \%$ at 1 year, $76 \%$ at 3 years and $62 \%$ at 5 years. Details of subsequent substance misuse post LT were available for 18/29 patients: 9/18 (50\%) reported illicit drug use. Return to illicit drug use after LT was not associated with reduced survival $(66 \%$ vs $78 \%$; $>0.05)$.

Conclusion To date only a minority of patients assessed in the UK on ORT have undergone LT (29/75), however outcomes are acceptable given the indications. The introduction of guidelines has improved the rates of formal drug misuse assessment and while return to substance misuse is common, this does not adversely affect survival.

\section{Competing interests None.}

Keywords liver transplantation, methadone, opiate replacement therapy. 